\title{
Rolle von miRNAs bei Biogenese und Funktion von Thrombozyten
}

Thrombozyten sind entscheidend an der primären Hämostase beteiligt, sie spielen aber auch eine Rolle bei verschiedenen Krankheiten. Dazu gehören das akute Koronarsyndrom sowie der Schlaganfall. Thrombozyten besitzen weder einen Kern noch genomische DNA. Allerdings finden sich in ihnen verschiedene RNA-Typen, darunter auch microRNAs (miRNAs). S. Dangwal und T. Thum fassten in einem Übersichtsartikel die wissenschaftlichen Ergebnisse zu miRNAs in Thrombozyten zusammen und diskutierten die Rolle dieser kurzen, hochkonservierten RNAs bei der Plättchenbiogenese und -funktion. Thromb Haemost 2012; 108: 599-604

Bei den miRNAs handelt es sich um 2123 Nukleotide lange, nicht kodierende RNAs. Sie sind an der Regulation von etwa $60 \%$ aller proteinkodierenden SäugetierGene beteiligt. Dies geschieht unter anderem durch Translationshemmung. Mehrere Studien aus der jüngeren Vergangenheit beschrieben die Expression von miRNAs innerhalb von Thrombozyten. Offenbar beeinflussen miRNAs die Biogenese von Thrombozyten sowie deren Funktion. An der Bildung von Megakaryozyten (Megakaryozytopoese), aus denen die Thrombozyten entstehen, sind unter anderem folgende miRNAs beteiligt: miR-10a, miR-130 sowie miR-155. Bei der Differenzierung der Megakaryozyten spielen wiederum die miRNAs
miR-34a sowie miR-150 eine Rolle. Hyperreaktivität von Thrombozyten zeigt ein erhöhtes Risiko für einen Myokardinfarkt, Schlaganfall sowie für eine periphere Arterienkrankheit an, während eine Hyporeaktivität die Blutungsneigung erhöht. Mehrere Studien untersuchten jüngst die Assoziation zwischen miRNAs und der Reaktivität von Thrombozyten. So steht die miR-96a in Zusammenhang mit der Granula-Exozytose, und miR-200b, miR-495 sowie miR-107 haben einen Einfluss auf die Hyperreaktivität der Thrombozyten.

Seit der Entdeckung von miRNAs in Thrombozyten konnten verschiedene erkrankungsspezifische miRNA-Expres-

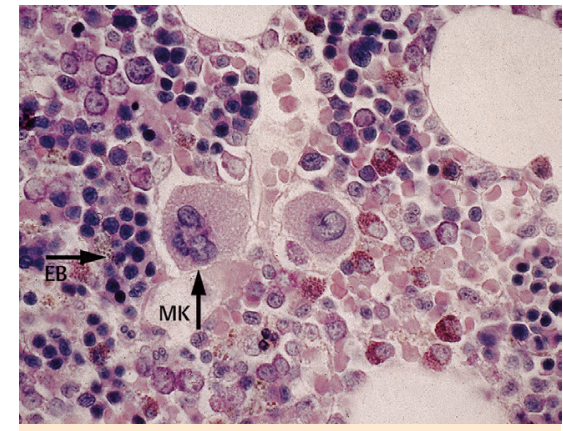

Knochenmark mit hyperplastischer Erythropoese. Die Abbildung zeigt viele Erythroblasten (EB) und einen Megakaryozyt, aus dem sich die Thrombozyten differenzieren in GiemsaFärbung (1:200) (Symbolbild, Quelle: Allgemeine und spezielle Pathologie. Riede, Werner, Schaefer, Hrsg., Stuttgart: Thieme Verlag; 2003).

sionsmuster ermittelt werden. So zeigten Thrombozyten von Patienten mit koronarer Herzkrankheit signifikant erhöhte Levels von miR-340* und miR-624*. Zudem war eine miR-28-Überexpression in Thrombozyten feststellbar, die von Patienten mit myeloproliferativen Neoplasien gewonnen wurden.

\section{Fazit \\ MiRNAs beeinflussen in vielfältiger Wei- se die Plättchenbiogenese und Plätt- chenfunktion. Zudem stehen Thrombo- zyten-miRNA-Expressionsmuster mit bestimmten Krankheitsbildern in $\mathrm{Zu}-$ sammenhang. Möglicherweise gelingt es zukünftig in Thrombozyten neue the- rapeutische miRNA-,,Targets“ für throm- botische bzw. kardiovaskuläre Erkran- kungen oder sogar für bestimmte Krebs- formen zu identifizieren, so die Hoff- nung der Autoren.}

\section{Dr. Frank Lichert, Weilburg}

